\title{
O Trabalho do Professor, Indicadores de Burnout, Práticas Educativas e Comportamento dos Alunos: Correlaçáo e Prediçáo ${ }^{1}$ THE Work of SPECIAL EDUCATION TEACHERS, BuRNOUT INDICATORS AND BEHAVIOR OF STUDENTS: CORRELATIONS AND PREDICTIONS
}

\author{
Nilson Rogério da SILVA² \\ Alessandra Turini BOLSONI-SILVA ${ }^{3}$ \\ Olga Maria Piazentin Rolim RODRIGUES ${ }^{4}$ \\ Vera Lúcia Messias Fialho CAPELLINI ${ }^{5}$
}

\begin{abstract}
RESUMO: a ocorrência do burnout em professores pode afetar também suas práticas educativas, seu repertório de habilidades sociais e o comportamento de alunos. Na literatura tais variáveis são abordadas de forma independente, o que dificulta sua compreensão. Este estudo teve como objetivos correlacionar e predizer as variáveis: condiçóes de trabalho do professor, indicadores de burnout, práticas educativas do professor e o repertório de habilidades sociais e de problemas de comportamento dos alunos. Participaram 94 professores do ensino regular de um curso de aperfeiçoamento (EAD), com turmas com a inserçáo de alunos com deficiência e/ou salas de recursos multifuncionais. Para a coleta de dados foram utilizados: 1) Questionário sobre a percepção dos professores sobre o trabalho docente, 2) Maslach Burnout Inventory - MBI, 3) Questionário de Habilidades Sociais Educativas para Professores e 4) Inventário de Comportamentos Pró-sociais. Os dados foram analisados com estatística descritiva (média e desvio padrão), correlação (Teste Spearman) e análises de regressão lineares. Os resultados indicaram que as práticas negativas influenciam o nível de burnout dos professores, o qual, por sua vez, é influenciado pelas condiçôes de trabalho, interferindo na saúde emocional do professor. Conclui-se para a importância de múltiplas medidas para avaliar trabalho e saúde do professor, bem como da necessidade de intervençóes que favoreçam melhores condições de trabalho e práticas educativas.
\end{abstract}

PALAVRAS-CHAVE: Educação Especial. Saúde do Professor. Burnout. Práticas Educativas.

ABSTRACT: The occurrence of burnout in teachers can also affect their educational practices, their repertoire of social skills and the behavior of students. In the literature, such variables are approached independently of each other, which makes it difficult to glean a full understanding of the issue. This study aimed to correlate and predict these variables: teacher working conditions, burnout indicators, educational practices of teachers, student repertoire of social skills and behavior. Ninety four regular education teachers, responsible for classes with students with disabilities or resource classroom who attended an improvement course participated in this study. Data was collected by applying: 1) a Questionnaire about teacher perceptions of the teaching profession; 2) the Maslach Burnout Inventory - MBI; 3) a Questionnaire of Educational Social Skills for Teachers; and 4) a Pro-social Behavior Inventory. Data were analyzed with descriptive statistics (mean and standard deviation), correlation (Spearman test) and linear regression analysis. Results indicated that negative practices influence the level of burnout of teachers, which, in turn, is influenced by working conditions, affecting the emotional health of teachers. The conclusion pointed to the importance of multiple measures to assess work and health of teachers, as well as the need for intervention promoting better working conditions and educational practices.

KEYWORDS: Special Education. Teacher health. Burnout. Educative Practices.

\footnotetext{
${ }^{1}$ http://dx.doi.org/10.1590/S1413-65382115000300004

${ }^{2}$ Programa de Pós-Graduação em Educação, Universidade Estadual Paulista - UNESP, Campus de Marília, Departamento de Fisioterapia e Terapia Ocupacional. SP, Brasil. nilson@marilia.unesp.br

${ }^{3}$ Programa de Pós-Graduação em Psicologia do Desenvolvimento, Universidade Estadual Paulista - UNESP, Campus de Bauru, Departamento de Psicologia, Bauru, SP, Brasil. bolsoni@fc.unesp.br

${ }^{4}$ Programa de Pós-Graduação em Psicologia do Desenvolvimento, Universidade Estadual Paulista - UNESP, Campus de Bauru, Departamento de Psicologia, Bauru, SP, Brasil. olgarolim29@gmail.com

${ }^{5}$ Programa de Pós-Graduação em Psicologia do Desenvolvimento, Universidade Estadual Paulista - UNESP, Campus de Bauru, Departamento de Educação, Bauru, SP, Brasil. vlmfcapellini@gmail.com
} 


\section{INTRODUÇÁo}

Os professores têm sido estudados em diferentes países (REINHOLD, 2007; TRIGO; TENG; HALLAK, 2007; POSIG; KICKUL, 2003), com variadas formas de investigação no que se refere ao impacto do exercício profissional no seu cotidiano de trabalho, considerando aspectos emocionais, físicos e organizacionais do ambiente e infraestrutura escolar.

Sobre o adoecimento do professor existe uma vasta literatura que aborda manifestações físicas como: problemas na voz (MARIANO; MUNIZ, 2006; PORTO et al., 2004); doenças musculoesqueléticas (SEIBT; LÜTZKENDORF; THINSCHMIDT, 2005; GASPARINI; BARRETO; ASSUNÇÃO, 2005); doenças respiratórias e cardiovasculares (PRIOSTE, 2006; GASPARINI; BARRETO; ASSUNÇÃO, 2005) e, cefaléia (MARIANO; MUNIZ, 2006).

Pode-se apontar ainda um conjunto de sinais e sintomas de doenças com componentes emocionais como os: indicadores de stress (REINHOLD, 2007; MARTINS, 2007; PRIOSTE, 2006; RODRIGUES et al., 2005; JAOUL; KOVESS, 2004); os transtornos psíquicos e de comportamento (BAUER et al., 2007; GASPARINI; BARRETO; ASSUNÇÃO, 2005) e os indicadores de burnout (BRAUN; CARLOTTO, 2014; CARLOTO, 2011; SILVA, 2009; SILVA; ALMEIDA, 2011; SCHWARZER; HALLUM, 2008; REIS et al., 2006).

Dentre os aspectos que podem levar ao adoecimento estáo aspectos do ambiente de trabalho: (a) físicos: temperatura, ventilação, iluminação, ruído e espaço físico (MARIANO; MUNIZ, 2006; LEVY, 2006); (b) organizacionais: jornada de trabalho extensa/sobrecarga de trabalho (ROCHA, 2008; MENDES, 2006); remuneração e reconhecimento (SAMPAIO; MARIN, 2004; LAPO; BUENO, 2003) e, (c) infraestrutura escolar: disponibilidade de materiais e recursos didáticos, adaptaçôes escolares, formação e capacitação de professores (BARASUOL, 2004; SILVA, 2009). Considerando tais variáveis parece que o contexto é desfavorável ao desempenho profissional do professor e para Oliveira (2005, p.1060) "A escola tem se mostrado um espaço gerador de tensôes e sofrimentos para os que nela trabalham".

Com base nesses estudos sobre o adoecimento físico e mental do professor pode-se dizer que a descrição desses problemas e de possíveis variáveis relacionadas torna-se importante. Dentre esses fenômenos o burnout tem sido um dos mais estudados em professores, sobretudo no que se refere aos aspectos organizacionais do ambiente de trabalho.

Segundo Posig e Kickul (2003), nas duas últimas décadas, o burnout foi abordado por diversos pesquisadores, principalmente ligado ao trabalho de profissionais da saúde e educação. O burnout pode ser entendido como um tipo especial de stress ocupacional crônico marcado pela presença de frustração e exaustão com o trabalho, cuja evolução é gradual e pode afetar todas as esferas da vida da pessoa (REINHOLD, 2007). Compreende três dimensōes: exaustão emocional; diminuição da realização pessoal e, despersonalização (MASLACH; JACKSON, 1981; PLANA; FABREGAT; GASSIÓ, 2003).

A incidência de burnout em vários países ainda é pautada na incerteza, mas dados sugerem um número significativo de indivíduos acometidos que pode variar entre $4 \%$ a $85,7 \%$, dependendo da população estudada (TRIGO; TENG; HALLAK, 2007). Em professores existem muitos estudos realizados no Brasil, mas os achados são variados. Carlotto (2011), por exemplo, encontrou a presença de alto nível de exaustáo emocional em 5,6\% de professores, 
despersonalização em $0,7 \%$ e baixa realização profissional em 28,9\%. Já no estudo de Codo (1999) realizado em âmbito nacional, com cerca de 39.000 trabalhadores da educação, encontrou em 25,1\% dos professores exaustão emocional, 31,9\% diminuição da realização pessoal e, em 10,7\%, despersonalização. Também não é incomum encontrar estudo em que o burnout não foi identificado em apenas uma destas dimensóes (REIS et al., 2006 ). Nesse sentido, reforçam os dizeres de Moura (1997) de que o burnout pode apresentar manifestaçóes e contornos específicos em função das singularidades presentes na organização e no ambiente de trabalho, bem como nas variáveis socioculturais.

Quanto à influência da sobrecarga laboral na saúde do profissional podem ser citados alguns estudos. Gil Monte e Peiró (1997) e Maslach, Schaufeli e Leiter (2001) apontam a sobrecarga laboral como um intenso fator para o aparecimento da síndrome de burnout, já que a alta exigência das demandas no contexto do trabalho, acompanhada com o tempo limitado para a realização das tarefas gerariam a exaustão emocional. Carlotto (2011) identificou, em professores, relaçóes entre variáveis demográficas e laborais, sendo mais prevalente em mulheres, com maior carga horária, maior número de alunos em sala e que atuam em escolas públicas como mais suscetíveis a desenvolver burnout. Para este autor esses resultados revelam preocupaçáo, uma vez que tais professores continuam nas salas de aula, muitas vezes, agravando seus quadros clínicos, com consequências para sua saúde e implicaçóes para o ensino-aprendizagem dos alunos.

Algumas pesquisas têm apontado que a forma como professores interagem (práticas educativas) com seus alunos podem favorecer ou dificultar o desenvolvimento emocional (habilidades sociais e problemas de comportamento) e/ou acadêmico dos alunos. Nessa direção o estudo das práticas educativas do professor na interação com os comportamentos dos alunos e com as condiçóes de trabalho e burnout do professor se faz necessário.

Habilidades sociais compóem uma área de pesquisa e intervenção cujo objetivo é promover o desempenho efetivo das pessoas nas interaçóes sociais em variados contextos (DEL PRETTE; DEL PRETTE, 1999, 2001). Habilidades sociais são comportamentos presentes na interação social, que sugerem o favorecimento de resolução de problemas, diminuição de conflitos e atuam na promoção, manutenção ou enriquecimento das interações sociais de forma satisfatórias e agradáveis (BOLSONI-SILVA, 2002; BOLSONI-SILVA; CARRARA, 2010).

No que se refere ao estudo de habilidades sociais em crianças pré-escolares e escolares, a literatura tem sido frequente em apontar uma relação inversa entre esse repertório e a presença de problemas de comportamento (BARRETO; FREITAS; DEL PRETTE, 2011; BOLSONI-SILVA; LOUREIRO, 2011; BOLSONI-SILVA; MARTURANO; LOUREIRO, 2011; BERRY; O'CONNOR, 2010; KIM et al., 2010; LEME; BOLSONI-SILVA, 2010).

Em relação às interações do professor, suas práticas podem ser positivas e/ou negativas e, para o aluno, essas podem ter como consequência a emissão de problemas de comportamento e/ou habilidades sociais, podendo estabelecer influência mútua em ambos (BOLSONISILVA et al. , 2013). Estes autores destacam que as práticas negativas utilizadas por professores são mais comuns diante de crianças com problemas de comportamento.

Nesse sentido, parece haver associação entre o uso de práticas negativas do professor e a ocorrência de comportamentos problema nos alunos, bem como as práticas positi- 
vas associadas à emissão de comportamentos socialmente habilidosos de alunos (CASTRO; BOLSONI-SILVA, 2008; BAKER; GRANT; MORLOCK, 2008). A prevalência de problemas de comportamento quanto ao gênero também se mostra de forma diferenciada, ainda que a presença de habilidades sociais ocorra em ambos. Bolsoni-Silva et al. (2013) identificaram que comportamentos habilidosos são mais freqüentes em meninas e problemas de comportamento em meninos.

A literatura destaca um conjunto de práticas emitidas por professores como promotoras do desenvolvimento das crianças no contexto escolar coma a habilidade de comunicação (FONSECA, 2012; HOWIE et al., 2010; UYSAL; ERGENEKON, 2010); a valorização de comportamentos habilidosos (BARNETT et al., 1996); a colocação de regras e limites (GOMES, 2003); ensino para resolver problemas (ALLEN; BLACKSTON, 2003); o suporte e acolhimento (GREENE; OLLENDICK, 1993); a afetividade (BAKER; GRANT; MORLOCK, 2008; KULLOK, 2002; RIBEIRO, 2010); a participação familiar (WEBSTER-STRATTON et al., 2008) e estímulo à criatividade e interação social (OLIVEIRA; WECHSLER, 2002).

Hipotetiza-se, a partir dos estudos mencionados, que as condiçóes de saúde dos professores podem interferir na forma como interage com seus alunos, o que, por sua vez, pode interferir no repertório de habilidades sociais e de problemas de comportamentos dos mesmos em sala de aula.

Nesse sentido, os estudos acima mencionados parecem indicar a associação entre as condiçôes de trabalho, a saúde do professor, as práticas educativas dos professores e a aprendizagem e problemas de comportamento dos alunos. Contudo, nota-se na literatura que tais assuntos são abordados de forma independente e/ou com correlação, em algum nível, de uma ou outra variável, o que limita a compreensão do problema de forma plena.

Dessa forma, o presente estudo tem como objetivos correlacionar e predizer as seguintes variáveis: condiçóes de trabalho do professor, indicadores de burnout, práticas educativas do professor e o repertório de habilidades sociais e problemas de comportamento dos alunos.

\section{Método}

\subsection{Participantes}

Participaram do estudo 94 professores do ensino regular que participavam de um curso de aperfeiçoamento na modalidade de ensino à distância (EAD) sobre práticas educativas em Educação Especial e Inclusiva. Em suas turmas havia a inserção de alunos com deficiência e que poderiam frequentar as salas de recursos multifuncionais, se disponíveis no contexto escolar.

\subsection{CARACTERIZAÇÁO DA AMOSTRA}

Dos 94 professores, 61\% dos participantes trabalham em classes regulares com inserção alunos com deficiência e 39\% atuam em sala de recursos multifuncionais. Quanto ao gênero, a maioria (96\%) era do sexo feminino. A média de idade era de aproximadamente 41 anos, variando entre 27 e 64 anos. A carga horária média de trabalho era de 37 horas por semana. A jornada dupla aparece em $37 \%$ dos casos, referindo-se a trabalho em mais de uma 
escola. O tempo médio na função era de 12 anos. Quanto ao estado civil, 62\% eram casados, $15 \%$ solteiros, $7 \%$ divorciados e $4 \%$ viúvos.

\subsection{INSTRUMENTOS}

Para a coleta foram utilizados quatro instrumentos:

1. Um questionário para levantar a percepção dos professores sobre fatores de riscos ocupacionais presentes no trabalho docente que aborda os seguintes aspectos: a) dados pessoais; b) dados ocupacionais (profissão, formação, tempo na função, tempo de atuaçáo com o alunado da educação especial); c) sintomas, afastamento e tratamentos decorrentes do exercício profissional; d) principais posturas adotadas durante o trabalho; e) condiçóes de trabalho (ambiente de trabalho, organização do trabalho, materiais e equipamentos, recursos pedagógicos); f) formação e treinamento oferecidos para o cuidado do aluno e cuidados pessoais; g) sintomas psicossociais presentes no trabalho (fatores não físicos); h) sugestóes para melhoria das condições de trabalho e manutenção da saúde; i) relaçóes entre as condiçóes de trabalho e o desempenho do professor; j) relaçóes entre as condiçôes de trabalho e saúde do professor e, k) relaçóes entre as condiçóes de trabalho e o aprendizado do aluno.

2. O protocolo Maslach Burnout Inventory - MBI (versão traduzida, adaptada e validada para a realidade brasileira por Tamayo (1997) para avaliar a presença de burnout junto aos professores.. Para a composição dos itens que compóem as escalas, a pontuação utilizada pelo autor foi alterada em relação à versão original que oferecia sete possibilidades de resposta. Neste são oferecidas 5 possibilidades, ou seja, de 1 a 5 ( 1 = nunca, 2 = algumas vezes ao ano, 3 = algumas vezes ao mês, $4=$ algumas vezes por semana e $5=$ diariamente).

3. Questionário de Habilidades Sociais Educativas para Professores que visa investigar a forma como o professor se comporta em relação a seus alunos. As questóes abordam a frequência de ocorrência dos comportamentos de: iniciar conversação (perguntas e respostas), expressar sentimentos positivos e negativos, estabelecer limites e manejar comportamentos de conflito na escola. Esse instrumento está disponível em ALMEIDA-VERDU et al., 2008, p.32-35). A construção realizada pela autora foi a partir de outro instrumento validado (BOLSONI-SILVA, 2009) que avalia o repertório de habilidades sociais de pais.

4. Inventário de Comportamentos Pró-sociais que investiga a ocorrência de comportamentos adequados e inadequados que o aluno apresenta em diferentes situaçóes de interação na sala de aula. O instrumento está disponível em (ALMEIDA-VERDU et al., 2008, p.35-37).

\subsection{Procedimentos de coleta de dados}

O presente projeto é parte de um projeto maior que foi aprovado pelo Comitê de Ética em Pesquisa da Faculdade de Ciências, da UNESP, Bauru/SP (processo n. 192/46/01/10). Os procedimentos para a realização da coleta de dados foram: 
- Contato com os participantes para apresentar os objetivos da pesquisa e solicitar a colaboração na realização do estudo.

- Publicação dos questionários no ambiente Teleduc (ensino à distância), juntamente com os termos de consentimento livre e esclarecido. Foram utilizados apenas os questionários respondidos acompanhados de assinatura do termo de consentimento livre e esclarecido devidamente preenchido.

\subsection{Procedimentos de tratamento E análise de dados}

Os dados foram analisados a partir de estatística descritiva (média e desvio padrão) para cada instrumento de forma a apresentar dados quantitativos e qualitativos da amostra estudada. Em um segundo momento foi computado os escores totais para cada um dos instrumentos, conforme instruçóes dos autores. Análises estatísticas de correlação (Teste Spearman) foram realizadas de forma a testar as hipóteses previstas no estudo relacionando as variáveis previstas. Os resultados foram organizados em tabelas.

Também foram conduzidas análises de regressão lineares. A primeira delas buscou identificar as variáveis preditivas de condição de trabalho, que é uma medida do instrumento de condição de trabalho/saúde; entraram no modelo as variáveis dos instrumentos de práticas educativas, problemas de comportamento/habilidades sociais infantis, burnout e as demais escalas do instrumento de condiçóes de trabalho/saúde (aspectos físicos e aspectos emocionais). As demais análises de regressão buscaram verificar o valor preditivo de todas as variáveis, já mencionadas, para cada uma das dimensôes do burnout (realização, exaustão emocional e despersonalização).

\section{Resultados e discussáo}

Esta seção apresenta os achados da correlação entre as medidas do estudo (Tabela 1) e os resultados de predição das mesmas (Tabela 2).

Tabela 1 - Resultados Teste de Correlação de Pearson

\begin{tabular}{|c|c|c|c|c|c|c|c|c|c|c|}
\hline & $\begin{array}{c}\text { Ex_- } \\
\text { emoc }\end{array}$ & Despers & Dim_realiz & Prat_pos & $\begin{array}{c}\text { Prat_ } \\
\text { neg }\end{array}$ & $\begin{array}{l}\text { Aspec_ } \\
\text { fisicos }\end{array}$ & $\begin{array}{c}\text { Aspec_ } \\
\text { emoc }\end{array}$ & $\begin{array}{c}\text { Cond_ } \\
\text { trab }\end{array}$ & $\begin{array}{c}\text { Comp. } \\
\text { Adeq }\end{array}$ & $\begin{array}{c}\text { Comp.- } \\
\text { inad }\end{array}$ \\
\hline Ext_emoc & & $0,425^{* *}$ & $-0,052$ & 0,007 & $0,222^{*}$ & 0,042 & 0,180 & $-0,125$ & $-0,130$ & 0,082 \\
\hline Desper & & & $0,335^{* *}$ & 0,105 & 0,053 & $-0,133$ & $-0,032$ & 0,014 & 0,045 & $-0,140$ \\
\hline Dim_realiz & & & & 0,038 & $-0,153$ & $-0,058$ & $-0,222^{*}$ & $0,262^{*}$ & 0,134 & $-0,215^{*}$ \\
\hline Prat_pos & & & & & $0,325^{* *}$ & $-0,025$ & 0,073 & 0,017 & 0,071 & 0,007 \\
\hline Prat_neg & & & & & & $-0,010$ & 0,112 & $-0,088$ & $-0,080$ & $-0,024$ \\
\hline Aspec_fisicos & & & & & & & $0,581^{* *}$ & $-0,195$ & $-0,280^{* *}$ & 0,044 \\
\hline Aspec_emoc & & & & & & & & $-0,298^{* *}$ & $-0,242^{*}$ & $0,217^{*}$ \\
\hline Cond_trab & & & & & & & & & $-0,030$ & $-0,051$ \\
\hline Comp. Adeq & & & & & & & & & & $-0,453^{* *}$ \\
\hline Comp._inad & & & & & & & & & & \\
\hline
\end{tabular}

Fonte: elaboração própria. 
$\mathrm{Na}$ Tabela 1 é possível observar correlaçóes positivas e negativas. As correlaçóes positivas encontradas foram entre: Exaustão Emocional e Despersonalização; Exaustão Emocional e Práticas Negativas, Despersonalização e a Diminuição da Realização Pessoal e Diminuição da Realização Pessoal e Condiçóes de trabalho. As correlaçóes negativas encontradas foram: Diminuição da Realização Pessoal e Aspectos Emocionais; Diminuição da Realização Pessoal e Comportamentos Inadequados dos alunos; Aspectos Físicos do contexto escolar e Comportamento Adequado dos alunos; Aspectos Emocionais e Condiçóes de trabalho; Aspectos Emocionais e Comportamento Adequado; Comportamento Adequado e Comportamento Inadequado dos alunos.

A Tabela 2 apresenta as regressóes considerando as variáveis preditivas de condição de trabalho/saúde e as condizentes às medidas de burnout do professor (realização, exaustão emocional e despersonalização).

Tabela 2 - Regressóes Logísticas Lineares considerando as categorias relativas às variáveis preditivas de condições de trabalho (1), realização (2), exaustão emocional (3), e despersonalização.

\begin{tabular}{lcccccc}
\hline \multicolumn{7}{c}{ I.C. 95\% } \\
\hline & Odds radio & Inferior & Superior & $p$ & Tolerância & VIF \\
\hline Realizaçáo & 0,068 & 0,002 & 0,133 & 0,043 & 0,951 & 1,052 \\
Aspectos emocionais & $-0,132$ & $-0,236$ & $-0,028$ & 0,014 & 0,951 & 1,052 \\
\hline \multicolumn{7}{c}{$2-$ realizaçáa } \\
\hline Exaustáo emocional & $-0,249$ & $-0,508$ & 0,010 & 0,059 & 0,802 & 1,247 \\
Despersonalização & 1,033 & 0,521 & 1,544 & 0,000 & 0,814 & 1,228 \\
Condiçóes trabalho & 0,701 & 0,129 & 1,272 & 0,017 & 0,979 & 1,022 \\
\hline \multicolumn{7}{c}{$3-$ exaustão emocional } \\
\hline Despersonalizaçáo & 0,479 & 0,568 & 1,339 & 0,000 & 0,877 & 1,140 \\
Realizaçáo & $-0,186$ & $-0,307$ & 0,007 & 0,062 & 0,859 & 1,164 \\
Práticas negativas & 0,168 & $-0,031$ & 0,677 & 0,073 & 0,964 & 1,037 \\
\hline
\end{tabular}

Fonte: elaboraçáo própria.

A Tabela 2 mostra que as variáveis que foram preditivas das condições de trabalho a realização profissional e os aspectos emocionais, foram obtidas pelo instrumento que avaliou burnout e de condiçóes de trabalho/saúde, respectivamente. Portanto, as práticas educativas e os comportamentos infantis não predizeram as condiçóes de trabalho.

A escala de burnout possui três dimensóes as quais foram submetidas à análise de regressão. Na dimensão de despersonalização nenhuma variável de práticas, trabalho e comportamentos infantis foram preditivas. Para a dimensão de realização profissional, as outras duas escalas de burnout (exaustão emocional e despersonalização) e as condições de trabalho a predizeram. Considerando a dimensão de exaustão emocional houve predição das outras duas dimensóes de burnout e das práticas educativas.

Com base nestes resultados pode-se concluir que as práticas negativas influenciam o nível de burnout dos professores, o qual é influenciado pelas condições de trabalho. Estas, por sua vez, têm influência da realização (escala burnout) e dos aspectos emocionais do professor. 


\section{Discussáo}

Conforme mencionado anteriormente, foi encontrado um conjunto de correlaçóes entre as variáveis estudadas, cuja discussão é apresentada a seguir:

\subsection{Correlaçóes positivas}

\subsubsection{ExaUstáo emocional e desPersonalização}

A correlação entre exaustão emocional e despersonalização indica que a presença de fatores de sobrecarga pode favorecer o processo de despersonalização. Gil Monte e Peiró (1997) e Maslach, Schaufeli e Leiter (2001) encontraram correlação entre exaustão (elevada demanda de trabalho e tempo insuficiente para realizar as atividades) e a incidência de burnout.

O início de um trabalho, geralmente, é marcado de satisfação e engajamento e, desta forma, é menos provável que o burnout ocorra nesta fase, sendo a transformação dos sentimentos gradativamente substituídos por comportamentos e atitudes negativas (MASLACH; LEITER, 1997). A presença da exaustáo emocional nos professores pode desencadear prejuízos na preparação e planejamento de aulas, diminuição ou perda da motivaçáo, na criatividade, na empatia, gerando um processo de distanciamento na relaçáo com os alunos (CARLOTTO, 2011).

Reis et al. (2006) em estudo com professores da rede municipal de Vitória da Conquista identificaram como frequentes a presença de cansaço mental e nervosismo e que tais fatores de riscos constituem elementos para as manifestaçóes de exaustão emocional.

Vale destacar que a exaustáo emocional é considerada uma das primeiras manifestaçôes do burnout da qual podem advir outros sintomas como é o caso da despersonalização e diminuição da realização pessoal (CODO, 1999; BENEVIDES-PEREIRA, 2002).

\subsubsection{EXAUSTÁo EMOCIONAL E PRÁTICA NEGATIVA}

A exaustáo emocional também apresentou correlação com a prática negativa. Comportamentos e atitudes negativas dos professores podem ser comuns em situação de desgaste emocional e, assim, afetar as relaçóes entre professores e alunos. Lyra et al. (2009) identificou a presença de sofrimento psíquico em $28 \%$ dos professores. Observaram, ainda, que professoras com sofrimento psíquico apresentaram de forma mais frequente problemas internalizantes (ansiedade e depressão) em seus alunos $(15,2 \%)$ quando comparadas àquelas que não manifestaram transtorno (7,3\%). Nesse sentido, indicam que o estado de saúde de professores interfere o julgamento dos comportamentos dos alunos e pode influenciar as práticas educativas.

A associação entre o uso de práticas negativas do professor e a presença de problemas comportamentais nos alunos tem sido extensamente descrita na literatura (CASTRO; BOLSONISILVA, 2008; BAKER; GRANT; MORLOCK, 2008; BOLSONI-SILVA et al., 2013). 


\subsubsection{DesPersonalizaÇáo E DIMINUIÇáo da REALIZAÇÁO PESSOAL}

Também foi observada correlação entre a despersonalização e a diminuição da realização pessoal. Como consequência da falta de ânimo e energia, o indivíduo desenvolve um afastamento psíquico e emocional dos alunos e de suas relaçóes, podendo atingir inclusive suas relaçóes sociais.

Volpato et al. (2003) apontam a despersonalização como uma dimensão característica da síndrome, que a diferencia do stress. Apresenta-se como uma maneira do profissional defender-se da carga emocional que ocorre devido ao contato direto com outras pessoas. Dessa forma, o profissional desenvolve atitudes insensíveis em relação às pessoas no trabalho, defendendo-se de possíveis repercussóes dos problemas e sofrimentos alheios em sua vida.

A situação se agrava quando, somada ao aspecto exaustáo emocional, a despersonalização leva ao comprometimento do desempenho e sensação de incompetência estabelecendo-se, assim, a redução do sentimento de realização profissional ou ainda o baixo envolvimento pessoal no trabalho.

Nessa condição, o trabalhador perde o sentido de sua relação com o trabalho (VASQUES-MENEZES; CODO, 2006) podendo favorecer ideias de abandono da profissão (REINHOLD, 2007).

\subsubsection{Diminuiçấo da REALIZAÇÁo PESSOAL E CONDIÇÓES DE TRABALHO}

Silva e Carlotto (2003) descrevem que o cotidiano dos professores é permeado por condiçóes de trabalho adversas, baixos salários, insuficiência de recursos materiais e didáticos, classes superlotadas, tensão no relacionamento com os alunos, carga horária excessiva, inexpressiva participação no planejamento da instituição e nas políticas institucionais e falta de segurança no ambiente escolar. Tais condiçóes podem interferir na saúde dele e no julgamento de sua capacidade e da qualidade do seu trabalho. Segundo Maslach e Jackson (1981) na fase de diminuiçấo da realização pessoal são comuns sentimentos de frustração, arrependimento e insatisfação, com julgamento negativo de si próprio. Ocorre falta de motivação para seguir em frente com seu trabalho e tornam-se presentes sensaçóes de menor rendimento, insatisfação com o seu desenvolvimento profissional e um sentimento de inadequaçáo no trabalho. A autoestima e autoconfiança ficam prejudicadas. Peeters e Rutte (2005) identificaram que a combinação de alta demanda de trabalho e baixa autonomia são fatores preditivos para conduzi-los ao burnout, sobretudo com a presença de exaustão emocional e implicações na dimensão da realização pessoal.

Nesse sentido, os dados revelam que as condições de trabalho podem favorecer o adoecimento de professores (presença do burnout, em todas as suas dimensóes) e influenciar as suas habilidades estimulando práticas educativas negativas. Por conseguinte, essas práticas favorecem dificuldades de aprendizagem e problemas de comportamentos nos alunos, afetando a saúde dos professores.

De maneira inversa, conforme as correlaçóes negativas encontradas, os dados revelam que quanto melhores as condiçóes de trabalho, menor a incidência de adoecimentos físicos ou 
emocionais, maior a satisfação e sentimento de realização com o trabalho e, consequentemente, menos frequentes os indicadores de problemas de comportamento nos alunos.

\section{CONSIDERAÇÓES FINAIS}

Desta forma, pode-se concluir que o exercício profissional de professores é permeado por situações que oferecem riscos à saúde física e emocional decorrentes das condiçóes existentes no ambiente e infraestrutura escolar, dos aspectos relativos à organização do trabalho, do sistema de ensino, das transformaçóes resultantes da reforma no setor educacional e do repertório de habilidades sociais do professor. Na mesma direção, a atuação do professor, dependendo da qualidade das relaçóes interpessoais e, consequentemente, do repertório de habilidades sociais e educativas, pode interferir negativamente no aprendizado do aluno e no desenvolvimento.

Assim, os resultados do presente estudo revelam a existência de correlaçóes entre as condiçôes de trabalho e infraestrutura escolar que pode favorecer o adoecimento de professores (físico ou mental). O adoecimento pode interferir na avaliação que o professor faz do aluno quanto à presença de comportamentos inadequados (avaliação negativa). Dessa forma, o repertório de práticas educativas interfere no comportamento e aprendizagem dos alunos. Nesse sentido, esta investigação apresenta uma possibilidade de estudar os assuntos de forma ampla, considerando as variáveis como multidirecionais.

Os dados também sugerem a necessidade de programas de intervençôes com aos professores, seja em benefício da sua própria saúde ou no desenvolvimento de estratégias de intervenção junto aos alunos, com benefício para ambos. Contudo, os programas de intervenção tem sido escassos ou praticamente inexistentes (HERNÁNDEZ et al., 2002). Alguns estudos mostraram efetividade (a partir do que?) para enfrentamento do burnout (SCHWARZER; HALLUM, 2008; FRIEDMAN, 2003; DORMAN, 2003; HOWARD; JOHNSON, 2004). $\mathrm{Na}$ mesma direção programa de intervençóes junto a professores para o desenvolvimento de habilidades sociais e práticas educativas tem mostrado resultados positivos (CONROY et al., 2014; BOLSONI-SILVA; CARRARA, 2010). Assim, programas que contemplem esses conteúdos parece ser um caminho para enfrentamento dos problemas no contexto escolar.

A amostra estudada permitiu resultados de predição e não apenas de correlação, o que representa um ganho metodológico. Contudo, recomenda-se que novos estudos sejam realizados com professores com a aplicação dos instrumentos de forma presencial e não online como no presente estudo. Outro aspecto importante seria incluir na coleta instrumentos de avaliação da saúde mental de professores.

\section{REFERÊNCIAS}

ALLEN, S. J.; BLACKSTON, A. R. Training preservice teachers in collaborative problem solving: An investigation of the impact of teacher and student behavior change in real-world settings. School Psychology Quarterly, v.18, n.1, p.22-51, 2003. 
ALMEIDA-VERDU, A.C.; RODRIGUES, O.M.P.R.; CAPELLINI, V.L.M.F. Repensando a avaliação. In: CAPELLINI, V.L.M.F.; RODRIGUES, O.M.P.R. (Org.). Práticas em educação especial e inclusiva na área da deficiência mental. Bauru: MEC/FC/SEE, 2008. v.12.

BAKER, J. A.; GRANT, S.; MORLOCK, L. The teacher-student relationship as a developmental context for children with internalizing or externalizing behavior. School Psychology Quarterly, v.23, n.1, p.3-15, 2008.

BARASUOL, E. B. Burnout docente no trabalho com a inclusão de alunos com necessidades educacionais especiais. 2004. Dissertação (Mestrado) - Programa de Pós-Graduação em Educação da Universidade Federal de Santa Maria, Santa Maria, 2004.

BARNETT, D. W. et al. keystone targets for change: planning for widespread positive consequences. School Psychology Quarterly, v.11, n.2, p.95-117, 1996.

BAUER, J. et al. Working conditions, adverse events and mental health problems in a sample of 949 German teachers. Int Arch Occup Environ Health, v.80, p.442-449, 2007.

BENEVIDES-PEREIRA, A. M. T. Burnout: quando o trabalho ameaça o bem-estar do trabalhador. 1. ed. São Paulo: Casa do Psicólogo, 2002.

BOLSONI-SILVA, A. T. Habilidades sociais educativas, variáveis contextuais e problemas de comportamento: comparando pais e mães de pré-escolares. 2003. Tese (Doutorado) - Universidade de São Paulo, Ribeirão Preto, 2003.

BOLSONI-SILVA, A. T. Estudos de confiabilidade e de validade do roteiro de entrevista de habilidades sociais educativas parentais (RE-HSE-P). 109f. 2009. Tese (Pós-Doutorado) - Faculdade de Medicina, Universidade de São Paulo, Ribeirão Preto, 2009.

BOLSONI-SILVA; A.T. et al. Contexto escolar: práticas educativas do professor, comportamento e habilidades sociais infantis. Revista Brasileira de Psicologia Escolar e Educacional, São Paulo, v.17, n.2, p.259-269, 2013.

BOLSONI-SILVA, A. T.; CARRARA, K. Habilidades Sociais e análise do comportamento: compatibilidades e dissensóes conceitual-metodológicas. Psicologia em Revista, v.16, p.330-350, 2010.

BOLSONI-SILVA, A. T.; LOUREIRO, S. R. Validação do roteiro de entrevistas de habilidades sociais educativas (RE-HSE-P). Avaliação Psicológica, v. 9, n.1, p.63-75, 2011.

BOLSONI-SILVA, A. T.; LOUREIRO, S. R.; MARTURANO, E. Validação do questionário de respostas socialmente habilidosas versão para professores - QRSH-Pr. The Spanish Journal of Psychology, v.29, n.2, p.227-234, 2011.

CARLOTTO, M. S. A síndrome do burnout e o trabalho docente. Psicologia em Estudo, Maringá, v.7, n.1, p.21-29, 2002.

CARLOTTO, M. S. Síndrome de Burnout em professores: prevalência e fatores associados. Psicologia: Teoria e Pesquisa, Brasília, v.27 n. 4, p.403-410, 2011.

BRAUN, A. C.; CARLOTTO, M. S. Síndrome de Burnout : estudo comparativo entre professores do ensino especial e do ensino regular. Revista da Associação Brasileira de Psicologia Escolar e Educacional, Sáo Paulo, v.18, n.1, p.125-133, 2014.

CASTRO, A.B.; BOLSONI-SILVA, A.T. Habilidades sociais na educação: relação entre concepções e práticas docentes na educação infantil. In: CAPELLINI, V.L.M.F. (Org.). Políticas públicas, práticas 
pedagógicas e ensino-aprendizagem: diferentes olhares sobre o processo educacional. Bauru: Cultura Acadêmica, 2008.

CODO, W. (Org.). Educação: carinho e trabalho. 4.ed. Petrópolis: Vozes, 1999.

DEL PRETTE, Z.A.P. ; DEL PRETTE, A. Psicologia das relaçóes interpessoais. Vivências para o trabalho em grupo. Petrópolis: Vozes, 2001.

CONROY, M. et al. Early childhood teachers' use of effective instructional practices and the collateral effects on young children's behavior. Journal of Positive Behavior Interventions, v.16, n.2, p.81-92, 2014.

DORMAN, J.P. Relationship between school and classroom environment and teacher burnout: a LISREL analysis. Social Psychology of Education, v.6, p.107-127, 2003.

FONSECA, B.C.R. Práticas educativas de genitores e professoras e repertório comportamental de crianças do ensino fundamental: estudos de caso. 2012. Dissertação (Mestrado em Psicologia) - Programa de Pós-Graduação em Psicologia do Desenvolvimento e Aprendizagem, Universidade Estadual Paulista, São Paulo, 2012.

FRIEDMAN, I. A. Self-efficacy and burnout in teaching: the importance of interpersonal-relations efficacy. Social Psychology of Education, v.6, p.191-215, 2003.

GASPARINI, S.M.; BARRETO, S.M.; ASSUNÇÃO, A.A. O professor, as condiçôes de trabalho e os efeitos sobre sua saúde. Educação e Pesquisa, São Paulo, v.31, n.2, p.189-199, 2005.

GIL-MONTE, P; PEIRÓ, J.M. Desgaste psíquico en el trabajo: el síndrome de quemarse. Madrid, Síntesis, 1997.

GOMES, L. Trabalho multifacetado de professores/as: a saúde entre limites. 2002. Dissertação (Mestrado) - Escola Nacional de Saúde Pública - ENSP Fundação Oswaldo Cruz - FIOCRUZ, 2002.

GREENE, R.W.; OLLENDICK, T.H. Evaluation of a multidimensional program for sixth-grader in transition from elementary to middle school. Journal of Community Psychology, v.21, p.162-176 1993.

HERNÁNDEZ, E.G. et al. Prevenção e intervenção na síndrome de burnout. Como prevenir ou remediar o processo de burnout. In: BENEVIDES-PEREIRA, A.M.T. Burnout: quando o trabalho ameaça o bem-estar do trabalhador. 1. ed. Sáo Paulo: Casa do Psicólogo, 2002. p.227-271.

HOWIC, L.D. et al. Participação em atividades fora do horário escolar em relação aos problemas de comportamento e habilidades sociais na infância, J. Health SCH, v.80, p.119-125, 2010.

JAOUL, G.; KOVESS, V. Le burnout dans la profession enseignante. Annales Médico Psychologiques, v.162, p.26-35, 2004.

KULLOK, M.G.B. (Org.). Relação professor-aluno: contribuições à prática pedagógica. Maceió: INEP, 2002.

LAPO, F.R.; BUENO, B.O. Professores, desencanto com a profissão e abandono do magistério. Caderno de Pesquisa, n.118, p.65-88, 2003.

LYRA, G.F.D. et al. A relação entre professores com sofrimento psíquico e crianças escolares com problemas de comportamento. Ciência \& Saúde Coletiva, v.14, n.2, p.435-444, 2009.

MARIANO, M.S.S.; MUNIZ, H.P. Trabalho docente e saúde: o caso dos professores da segunda fase do ensino fundamental, Estudos e Pesquisas em Psicologia, Rio de Janeiro, v.6, n.1, p.6-88, 2006.

MARTINS, M.G.T. Sintomas de stress em professores brasileiros. Revista Lusófona de Educação, v.10, p.109-128, 2007. 
MASLACH, C.; JACKSON, S.E. Maslach bournout inventory. Human services survey. Palo Alto: Consulting Psychologics Press, 1981.

MASLACH, C.; SCHAUFELI, W.B.; LEITER, M.P. Job Burnout. Annual Review of Psychology, v.52, p.397-422, 2001.

MENDES, M.L.M. Condiçôes de trabalho e saúde docente. In: SEMINÁRIO DA REDESTRADO - REGULAÇĀO EDUCACIONAL E TRABALHO DOCENTE, 6., Rio de Janeiro, 2006. Anais... UERJ, Rio de Janeiro, 2006.

MOURA, E.P.G. Saúde mental e trabalho. Esgotamento profissional em professores da rede de ensino particular de Pelotas - RS. 1997. Dissertação (Mestrado) - Pontifícia Universidade Católica do Rio Grande do Sul, Porto Alegre, 1997.

OLIVEIRA, D.A. A reestruturação do trabalho docente: precarização e flexibilização. Educação e Sociedade. Campinas, v.26, n.92, p.753-775, 2005.

OLIVEIRA, D.L. Processo de trabalho e saúde na escola: $u \mathrm{~m}$ estudo de caso com professores do ensino fundamental da escola municipal General Mourão Filho em Duque de Caxias. 2001. Dissertação (Mestrado) - Escola Nacional de Saúde Pública, Rio de Janeiro, 2001.

OLIVEIRA, E.T.A.; WECHSLER, S. M. Variáveis que afetam a aprendizagem: percepção de alunos de licenciatura e professores. Psicologia Escolar e Educacional, v.6, n.2, p.133-139, 2002.

PEETERS, M.A.G.; RUTTE, C. G. Time management behavior as a moderator of the job demandcontrol interaction. Journal of Occupational Health Psychology, v.10, n.1, p.64-75, 2005.

PORTO, L.A. et al. Doenças ocupacionais em professores atendidos pelo Centro de Estudos da Saúde do Trabalhador - CESAT. Revista Baiana de Saúde Pública, v.28, n.1, p.33-39, 2004.

POSIG, M.; KICKUL, J. Extending our understanding of burnout: Test of integrated model in nonservice occupations. Journal of Occupational Health Psychology, n.8, n.1, p.3-19, 2003.

PRIOSTE, C.D. Diversidade e adversidades na escola. Queixas e conflitos de professores frente à educação inclusiva. 2006. Dissertação (Mestrado em Educação) - Faculdade de Educaçáo, Universidade de São Paulo, São Paulo, 2006.

REINHOLD, H.H. O Burnout. In: LIPP, M.E.N. (Org.). O stress do professor. 5.ed. São Paulo: Papirus, 2007. p.63-80.

REIS, E.J.F.B. et al. Docência e exaustão emocional. Educação Sociedade, Campinas, v.27, n.94, p.229253, 2006.

RIBEIRO, M. L. A afetividade na relaçâo educativa. Estudos de Psicologia, Campinas, v.27, n.3, p.403412, 2010.

ROCHA, E.K.G.T. Desigualdade também no adoecimento: mulheres como o alvo preferencial das síndromes do trabalho. In: ENCONTRO NACIONAL DE ESTUDOS POPULACIONAIS,16., 2008, Caxambu. Anais..., 2008.

RODRIGUES, M.L. et al. Estresse ocupacional: um estudo com professores das redes pública e privada de ensino. Revista da Sociedade de Psicologia do Triângulo Mineiro - SPTM, Uberlândia, v.9, n.1, p.37- 44, 2005.

SAMPAIO, M.M.F.; MARIN, A.J. Precarização do trabalho docente e seus efeitos sobre as práticas curriculares. Educação e Sociedade, Campinas, v.25, n.89, p.1203-1225, 2004. 
SCHWARTZ, Y. la "Qualification" à la "Compétence". Education Permanente, n.123, p.125-138, 1995.

SEIBT, R.; LÜTZKENDORF, L.; THINSCHMIDT, M. Risk factors and resources of work ability in techers and office workers. International Congress Series, v.1280, p.310-315, 2005.

SILVA, G.N.; CARLOTTO, M.S. Síndrome de Burnout: um estudo com professores da rede pública. Revista de Psicologia Escolar e Educacional, v.7, n.2, p.145-153, 2003.

SILVA, N.R. Condiçôes de trabalho e saúde de professores de alunos com e sem necessidades educacionais especiais. 2009. Tese (Doutorado em Educação Especial) - Universidade Federal de São Carlos, Programa de Pós-Graduação em Educação Especial, São Carlos, 2009.

SILVA, N.R.; ALMEIDA, M.A. As características dos alunos são determinantes para o adoecimento de professores - um estudo comparativo sobre a incidência de Burnout em professores do ensino regular e especial. Revista Brasileira de Educação Especial, Marília, v.17, n.3, p. 373- 394, 2011.

TAMAYO, M.R. Relação entre a sindrome de Burnout e os valores organizacionais no pessoal de enfermagem de dois hospitais públicos. 1997. Dissertação (Mestrado em Psicologia) - Instituto de Psicologia, Universidade de Brasília, Brasília, DF, 1997.

UYSAL, A.; ERGENEKON, Y. Social skills instruction carried out by teachers working at private special education institutions in Turkey. Education and training in autism and developmental disabilities, v.45, n.3, p.459-466, 2010.

VASQUES-MENEZES, I; CODO, W. O que é burnout. In: CODO, W. (Org.). Educaçâo: carinho e trabalho. 3.ed. Petrópolis: Vozes, 2002. p.237-254

VOLPATO, D.C. et al. Burnout: O desgaste dos professores de Maringá. Revista Eletrônica InterAção Psy, v.1, n.1, p.90-101, 2003.

WEBSTER-STRATTON, C.M.; REID, J.; STOOLMILLER, M. Preventing conduct problems and improving school readiness: Evaluation of the incredible years teacher and child training programs in high-risk schools. Journal of Child Psychology and Psychiatry, v.49, n.5, p.471-488, 2008.

Recebido em: 12/09/2014

Reformulado em: 16/05/2015

Aprovado em: 16/05/2015 